\title{
4.3 Die Beurteilung eines Groß- projektes aus der Sicht einer Region am Beispiel der Neuen Haupttransversalen
}

\subsubsection{Die Region Burgdorf}

Seit 1966 besteht ein Zusammenschluß von heute 40 Gemeinden der Amtsbezirke Burgdorf, Fraubrunnen und Trachselwald, der sich die Durchführung der Regionalplanung zum Ziele gesetzt hat. Die Region umfaßt ein Gebiet von knapp 40000 ha mit 64500 Einwohnern am mittleren und unteren Lauf der Emme. Sie ist geprägt durch die Hügellandschaft südlich der Linie Wynigen-Burgdorf-Krauchthal und die weiten Ebenen der bernischen Kornkammer nördlich dieser Linie. Das Rückgrat der Region bilden die Verbindungen entlang der Emme, mit Straße und Bahn (EBT). Quer durch die Region laufen die großen West-OstAchsen der Schweiz von Bern in Richtung Olten. Der ersten Straße über Burgdorf folgte noch im 18. Jh. die Straße über Kirchberg. Um 1850 wurde die Hauptlinie der Centralbahn auf Intervention (und Kostenbeteiligung) Burgdorfs entgegen der ursprünglichen $\mathrm{Ab}$ sicht nicht parallel zur Staatsstraße gebaut, sondern entlang der mittelalterlichen Achse über Burgdorf geführt. Die Nationalstraße N1 wurde über Kirchberg geführt, strebt dann allerdings nicht Olten zu, sondern holt nach Norden in Richtung Solothurn aus. Die Autobahn ist bei Alchenflüh an das regionale Straßennetz angeschlossen.

Die Ausgangs- und Zielpunkte des Fernverkehrs sind seit Jahrhunderten konstant, ebenso das Ringen um die Linienführung durch unsere Region. Der technisch besten Linie über Kirchberg wurde immer wieder politischer Druck entgegengesetzt, der das Zentrum Burgdorf einbeziehen wollte. Mit den Plänen einer NHT wird also nur ein grundsätzliches Problem erneut zur Diskussion gestellt.

Die Region Burgdorf verfügt als erste bernische Region seit 1977 über genehmigte Richtpläne nach dem Baugesetz von 1970. Die wichtigsten Ziele der Raumordnung können als langfristige Sicherung eines optimalen Lebensraumes für die eigene Bevölkerung bezeichnet werden. Der Siedlungsraum für Wohnen und Arbeiten wird streng begrenzt, damit möglichst viel Fläche der Landwirtschaft erhalten bleibt. Der intakte ländliche Raum mit traditioneller Nutzung und Baustruktur bildet die Grundlage für den hohen Erholungswert der Region. Die Verkehrsflächen haben sich, von einigen nötigen Ortsumfahrungen abgesehen, auf die heute bestehenden Anlagen'zu beschränken.

\subsubsection{Die Vorgeschichte}

Große nationale Projekte, wie die NHT, entstehen ja nicht plötzlich. Von der ersten Idee bis zum fertigen Projekt führt ein langer Entwicklungsweg. Für ein betroffenes Gebiet ist es sehr wichtig, möglichst früh Kenntnisse über solche Vorhaben zu erhalten, um die Auswirkungen im Raum abschätzen zu können, aber auch um Verbesserungsvorschläge in einem frühen Stadium einbringen zu können, wo Änderungen noch möglich sind. Die Region Burgdorf erfuhr erstmals über die Pläne der SBB, als bei der Grundlagenbearbeitung das damalige Studienbüro der GD SBB (Herr O. Baumann) befragt wurde. Da wir angehalten waren, die Information nicht direkt zu veröffentlichen, steht im Grundlagenbericht (GROSJEAN 1966/70) folgender Passus: "Laut Presseberichten (z. B. „Der Bund”, Nr. 37 vom 15. Februar 1970) wird für die 1980er Jahre das Projekt einer doppelspurigen Schnellbahnverbindung Bern-Rothrist enwogen, mit minimalen Kurvenradien von $4000 \mathrm{~m}$ und ohne Zwischenstationen, für Höchstgeschwindigkeiten von $300 \mathrm{~km} / \mathrm{h}$. Der genaue Linienverlauf ist nicht festgelegt, wird aber von Schönbühl in nordöstlicher Richtung den unteren Teil der Region Burgdorf durchschneiden." In der Personalzeitschrift von Emch + Berger (8/73) fanden wir erstmals eine Karte mit der Linienführung. Gleichzeitig wurde das Projekt der SBB den betroffenen Gemeinden vorgestellt, wobei ihm ein Gegenvorschlag der Region, der eine Führung entlang der N1 vorsah, entgegengehalten wurde. Die Reaktion der Gemeinden war vorauszusehen. Sie lehnten jeweils diejenige Variante ab, die ihr eigenes Terrain berührte. In Utzenstorf hatte die Vorstellung aber weitere Folgen. Es wurde ein brachliegender Fonds, der seinerzeit zum Kampf gegen ein Flughafenprojekt geschaffen wurde, reaktiviert und ein Komitee gegen die Schnellbahn gebildet. Im Entwurf zum regionalen Gesamtplan 1973 (Atelier 5, 1973) und in den Richtplänen 1976 (Atelier 5, 1976) wurde vom technischen Planer und vom Fachausschuß der Region einigermaßen positiv Stellung genommen: "Es handelt sich um ein übergeordnetes Planungsvorhaben, dessen Zweckmäßigkeit hier nicht zur Diskussion gestellt werden kann. Im heutigen

Charles Mäder, Dr., Geschäftsführer des Planungsverbandes Region Burgdorf, Bahnhofstrasse 43, 3400 Burgdorf 


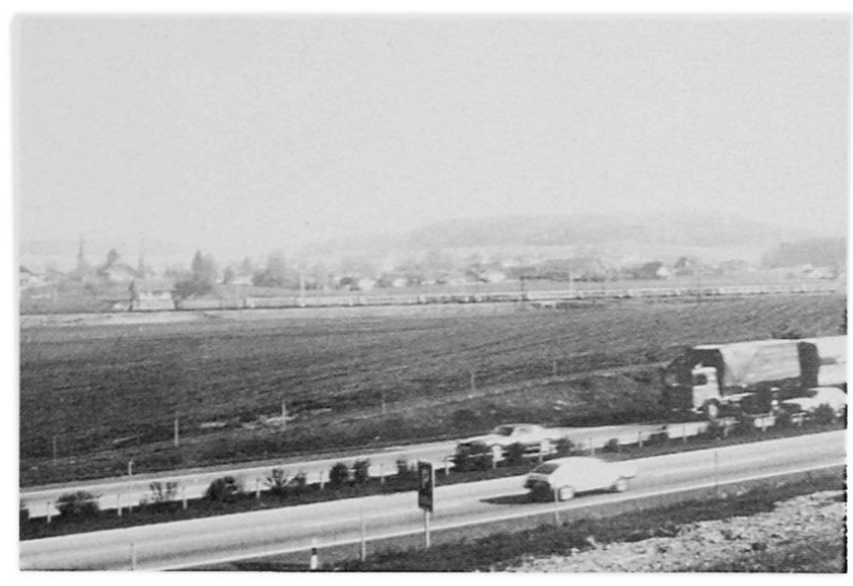

Aufnahme 1 (Ch. Mäder)

Das Projekt der SBB hätte zwischen heutiger Linie und N1 mit einem Damm von $14 \mathrm{~m}$ Höhe die Ebene schräg zerschnitten.

Zeitpunkt liegt der SBB-Vorschlag als eine technisch gut ausgearbeitete Lösung vor.» Die Gemeinden, welche zu den Richtplänen vor deren Genehmigung Stellung zu nehmen hatten, meldeten Bedenken an, und anläßlich einer Delegiertenversammlung wurde ein Antrag mit großem Mehr genehmigt, der die Region aufforderte, konsequent gegen die Schnellbahn Stellung zu nehmen. Die am 30.11.1977 in Kraft gesetzten Richtpläne enthalten deshalb einen neuen Passus: "Das SBBProjekt einer neuen Bahnlinie zwischen Bern und Olten würde der Landwirtschaft und der Landschaft der Region erhebliche Nachteile bringen, ohne der Region zu nützen. A us regionaler Sicht muß dieses Projekt deshalb abgelehnt werden." Der Kanton Bern meldete allerdings in seinem Prüfungsbericht zu den Richtplänen Bedenken an und behielt sich eine andere Beurteilung vor. Nach diesen internen Diskussionen der Region trat für einige Zeit Ruhe ein, bis die SBB das Projekt der Grauholzlinie (GD SBB, 1979) vorlegten.

\subsubsection{Die Grauholzlinie und Probleme der Information}

Seit der Einführung des Taktfahrplans zeigte sich sehr deutlich, daß die Strecke Zollikofen-Wilerfeld stark überlastet ist. An Stelle eines Ausbaus im Engnis von Zollikofen sehen die SBB den Bau einer neuen Linie unter dem Grauholz vor. 1979 wurde das Projekt den Gemeinden im Plangenehmigungsverfahren unterbreitet, ohne daß sie über die weiterführenden Pläne der NHT verfügten, die etwa gleichzeitig an die Regionen gingen. Der Planungsverband stellte bei einem Vergleich der Projekte fest, daß die Zustimmung zur Grauholzlinie bei einem eventuellen Bau der NHT untragbare Konsequenzen für die Gemeinde Hindelbank hätte.

Die weite, leicht hügelige Landschaft der Grundmoränen des eiszeitlichen $\mathrm{R}$ honegletschers erträgt keine langgestreckten bis $14 \mathrm{~m}$ hohen Dämme, wie sie das Projekt SBB vorsah. Das Landwirtschaftsgebiet der

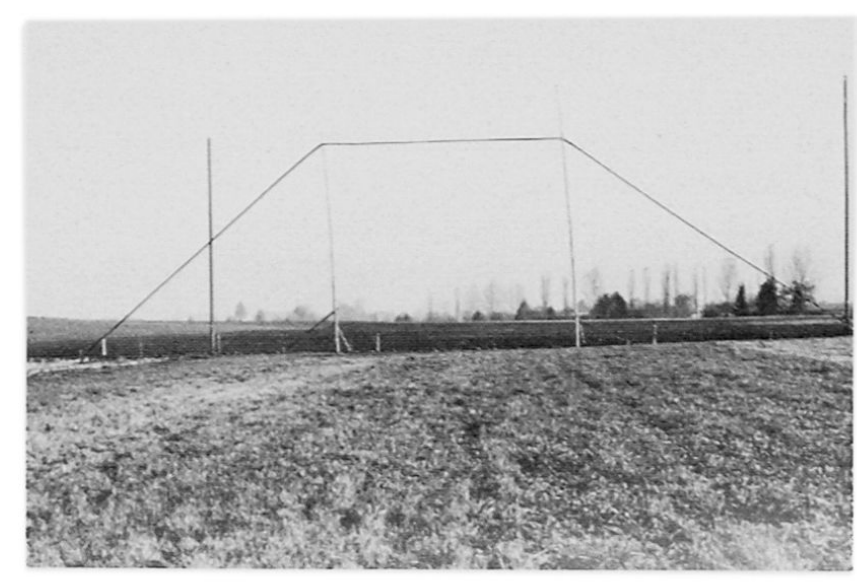

Aufnahme 2 (Ch. Mäder)

Das Profil des Dammes in der Ebene (Linien nachgezogen).

Gemeinde, das schon heute durch die bestehende Bahnlinie und die N1 zerschnitten wird, wäre erneut diagonal durchfahren worden (Bild 1). Es wären viele unwirtschaftliche Restflächen mit spitzen Winkeln entstanden. Die Gemeinde ließ durch ihr Personal ein Profil des Dammes aufgrund der Plandaten erstellen, damit die Folgen einer Zustimmung zur Grauholzlinie im Gelände sichtbar wurden (Bild 2). Das Profil wirkte derart überzeugend, daß der Direktor des Bundesamtes für Verkehr öffentlich diese Variante der SBB ablehnte.

Damit wird ein grundsätzliches Informationsproblem deutlich: Wenn der Träger eines Projektes (hier die GD SBB) den Betroffenen nur Teile vorstellt, so können sie sich keinen Überblick verschaffen, besonders wenn weitere Etappen ihnen vorenthalten werden. Der Vorwurf der "Salamitaktik» liegt in solchen Fällen nahe. Es ist deshalb zu fordern, daß die Träger von Großprojekten in jedem Fall zuerst über das ganze Vorhaben orientieren, wenn möglich mit Hilfe regionaler Organisationen, bevor einzelne Teile (wenn auch rechtlich in einem anderen Verfahren) zum Entscheid gestellt werden.

\subsubsection{Der Variantenentscheid NHT oder KAP 1981}

Im August 1981 wurden den betroffenen Kantonen vom Bundesamt für Verkehr die Projekte NHT 1980 und Streckenausbau Rothrist-Hindelbank 1978/79 zum Variantenentscheid unterbreitet. Der Kanton Bern holte über die Regionen die Meinungen der Gemeinden ein. Neben den technischen Berichten standen Pläne 1: 5000 und Längenprofile zur Verfügung.

Für die Region Burgdorf stellte sich die Aufgabe, die Projekte selbst zu beurteilen und den Gemeinden mit Informationen behilflich zu sein. Es wurden überall auf Anfrage Ausstellungen der Pläne und öffentliche Vorträge organisiert, an denen mit Hilfe von Dias und Folien die lokalen Auswirkungen der Projekte gezeigt 
wurden. Die Stimmung der anschließenden Diskussionen, welche die Haltung der Gemeinden mitbestimmte, war meist eindeutig ablehnend, besonders weil noch jegliche Entscheide durch den Bund oder den Souverän fehlten.

Grundsätzlich stehen drei Möglichkeiten zur Erhöhung der Kapazitäten eines Verkehrsträgers zwischen zwei bekannten Endpunkten zur Verfügung:

1. Eine Neuanlage, die sich nur an den technischen Elementen orientiert und unabhängig von bestehenden Achsen gelegt wird. Diesen Weg beschritt das Projekt SBB 1973. Für die Region hätte dies bedeutet, daß großflächige, bereits mehrmals meliorierte Landwirtschaftsgebiete so zerschnitten worden wären, daß erneut eine Güterzusammenlegung und ein neues Erschließungsnetz nötig geworden wären. Ein Vorteil hätte nur darin bestanden, da $\beta$ die größtmögliche Entfernung von bestehenden Siedlungen möglich gewesen wäre. Die Folgen in unserer Landschaft erachteten wir aber von Anfang an als untragbar.

2. Eine Neuanlage, die sich möglichst an bestehende Verkehrsträger anlehnt. Dazu ist in der Region das Trasse der N1 geeignet. Die Elemente der Autobahn (Radien und Steigungen) erlauben es aber nicht, die vorgesehene Art Eisenbahn parallel zu bauen, abgesehen von den Schwierigkeiten, die sich im Bereich der Anschlüsse ergeben. Durch die unterschiedlichen Radien entstehen immer wieder sichelförmige Restflächen, die landwirtschaftlich nicht mehr nutzbar sind. Eine Reduktion der Radien und eine Erhöhung der Steigungen (bis zu 35 Promille wie beim TGV in Frankreich) wäre mit dem Konzept der SBB (lokbespannte Züge, gemischter Verkehr, nachts auch Güterzüge) nicht mehr vereinbar, und ein Konzept wie beim TGV ist für die Schweiz nicht zweckmäßig (kurze Distanzen, gemischter Verkehr, große Passagierzahlen).

3. Eine Erweiterung der Kapazität durch zwei weitere Geleise entlang der bestehenden Anlagen, mit lokalen Korrekturen. Diese Art des Ausbaus brächte in der Region große Schwierigkeiten, indem das bisherige Trasse z. T. in engen Tälern verläuft, in denen nur mit untragbar großen Erdbewegungen genügende Radien erreicht werden könnten.

Für die Region Burgdorf erwies sich die vorgeschlagene Führung des Projektes SBB 1980 als das kleinste Übel, die Restflächen fielen meist in den Wald oder wurden durch das Abtauchen der Bahn in Tieflage gemildert (Bild 3).

Als schwierig erwies sich die Formulierung von Alternativen zu einzelnen Streckenabschnitten. Wir entschlossen uns, Pläne und Profile mit den gleichen Elementen zu erstellen, wie sie von den SBB vorgelegt wurden. Auf eisenbahntechnische Einzelheiten mußten wir mangels Kenntnissen verzichten, aber die wichtigen Anliegen konnten so kompetent vertreten werden. Besonders bei der Diskussion um den

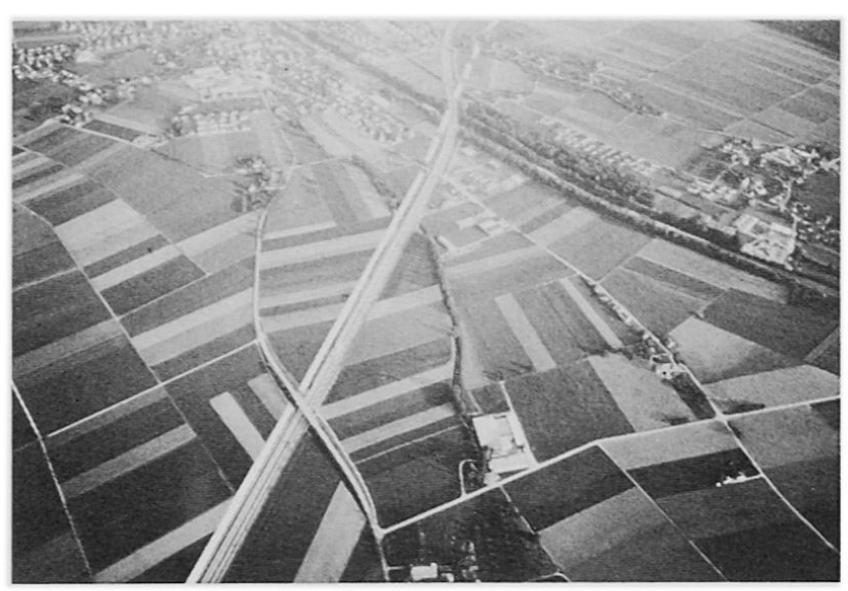

Aufnahme 3 (M. Wehrlin)

Die Führung des NHT-Projektes entlang der N1 im Raume Kirchberg mit Unterquerung der Emme, der N1, der EBT und der Siedlung.

Anschlußpunkt der Grauholzlinie erwiesen sich die Pläne als wertvoll, denn hier sollte nicht ein Projekt grundsätzlich abgelehnt, sondern eine bessere Lösung gefunden werden. Die SBB nahmen die Ideen der Region auf, und nach Beseitigung von Differenzen mit der Region Bern können wir heute mit Befriedigung feststellen, daß die oben erwähnten Probleme in Hindelbank gelöst werden konnten. Die Zerschneidung der Kulturflächen und der Damm werden vermieden, die Grauholzlinie beansprucht, vorausgesetzt $\mathrm{da} ß$ sie wie verlangt bis zur N1 überdeckt wird, wenig Kulturland, und eine mögliche NHT könnte entlang der Autobahn geführt werden.

Das Projekt der NHT sieht im Raume Kirchberg eine Unterquerung der Siedlungen, der $\mathrm{N} l$ und der Emme vor (Bild 3). Den Projektplänen lag ein Gutachten über die Auswirkungen auf das Grundwasser bei. Die Region hatte für ihre Stellungnahme zu beurteilen, ob die Auswirkungen tragbar wären. Durch die Tieflage wird zwar vermieden, $\mathrm{da} \beta$ die bestehenden und künftigen Siedlungsgebiete optisch und durch Lärm gestört würden, aber die Bauphase (offene Konstruktion) würde über einige Zeit starke Immissionen verursachen. Besonders schwierig war das Abschätzen der Auswirkungen auf den großen Grundwasserstrom im Fächer der Emmenebene. Nur die Beurteilung durch einen unabhängigen Fachmann brachte an den Tag, daß die Modellannahmen des Gutachtens falsch waren, wobei der Fehler im Modellsystem selbst lag. Wäre das Modell richtig, so müßten wir im Gebiet um Fraubrunnen einen respektablen See erhalten! Es ist für eine Region, die über ein Einmannsekretariat verfügt, sehr schwierig, in technischen Detailfragen über Projekte zu urteilen, die durch große Stäbe von Fachleuten erstellt wurden. Gute Kenntnisse der lokalen Einzelheiten und ein Verständnis für planerische und technische Fragen vorausgesetzt, ist es aber durchaus möglich, die Schwachpunkte der Projekte zu finden und entsprechend zu reagieren. 
Die einhellige Ablehnung der Varianten NHT und KAP durch die Gemeinden (ausgenommen die Stadt Burgdorf) bewog uns, der Delegiertenversammlung zu beantragen, weiterhin am Text der Richtpläne 1977 festzuhalten. Trotzdem wurde im ausführlichen Bericht der Kanton auf die regionalen und lokalen Schwierigkeiten aufmerksam gemacht. Ein Hauptpunkt war die Forderung nach Grundlagen über die Zweckmäßigkeit und Umweltverträglichkeit der Projekte. Wir hofften, mit diesem Bericht unsere grundsätzliche Meinung deutlich kundtun zu können, ohne uns von weiteren technischen Diskussionen auszuschließen.

\subsubsection{Die Zweckmässigkeitsprüfung der NHT}

Im Auftrage des Stabes für Gesamtverkehrsfragen erstellte 1982/83 die Arbeitsgemeinschaft Güller/Infras einen Bericht über die Zweckmäßigkeitsprüfung der neuen Haupttransversalen (NHT) (Zürich, 1982), der wiederum über den Kanton der Region und den Gemeinden zur Stellungnahme unterbreitet wurde. Im Gegensatz zu den teils hitzigen Diskussionen beim Variantenentscheid wurde von den Gemeinden meist lapidar festgestellt, man halte am Bescheid des Vorjahres fest. Für die Region selber stellte sich die Prüfung des Berichtes wesentlich schwieriger dar. Es mußte erstmals auf viele Fragen eingetreten werden, die den Rahmen unserer Betrachtungsweise sprengten. Aufgrund unserer Erfahrungen mit den bestehenden Projekten in unserer Region erlaubten wir uns, auch die übrigen Strecken unter den gleichen Gesichtspunkten kritisch zu betrachten. Es zeigte sich, daß ein Grundproblem der NHT in der Unvereinbarkeit der technischen Anforderungen (Radien, Steigungen) mit den Gegebenheiten unserer Landschaft (feingliedrige Topographie und Bodenbedeckung) liegt.

Auf unsicherem Boden bewegten wir uns bei der Beurteilung der Verkehrsentwicklung und der Wirtschaftlichkeit. Es ist einer Region nicht möglich, sich unabhängig relevante Fakten und Prognosen zu beschaffen, sei es wegen der hohen Kosten oder den zu kurzen Zeiten, die zur Verfügung stehen. Eingedenk dessen, daß auch die Verfasser der Zweckmäßigkeitsprüfung auf die Mitarbeit der wenigen Fachleute der SBB und des Bundes angewiesen waren, haben wir uns trotzdem erlaubt, die Annahmen der GVK über die Verkehrsentwicklung in Frage zu stellen, weil es uns nicht plausibel erscheint, daß das Verkehrsvolumen für Personen und Güter im überregionalen und internationalen Verkehr bei stagnierender Bevölkerung und immer rationeller werdender Technik noch derart zunehmen kann. Besonders gewagt schien uns die Wirtschaftlichkeitsberechnung, weil hier aus unbekannten Gründen nur die Folgen der NHT an sich, nicht aber die Auswirkungen auf das bestehende Netz betrachtet wurden.

Auch die Auswirkungen auf die gesamte Raumordnung des Mittellandes beurteilen wir anders als der
Bericht. Eine NHT würde u.E. die großen Agglomerationen der Schweiz weiter stärken und die zwischenliegenden Regionen schwächen, Gebiete, die im Kanton Bern zu den wirtschaftlich stabilsten gehören.

\subsubsection{Schlußfolgerungen}

Die Regionen in ihrer heutigen Form als Planungsvereine sind bei der Prüfung von Großprojekten in der Lage, einen Beitrag zu leisten. Dieser besteht in der internen Information der Gemeinden, in der gründlichen Analyse der Auswirkungen eines Projektes auf die Landschaft, die Siedlung, die Verkehrs- und Wirtschaftsstruktur innerhalb der Region. Dies gilt besonders, wenn eine Region über sorgfältig erarbeitete Richtpläne und Entwicklungsgrundsätze verfügt. Für die Beurteilung des Gesamtrahmens eines Projektes hingegen verfügt die Region über eine zu geringe personelle und finanzielle Basis. Ihr Beitrag kann erst bei sorgfältiger Wertung durch übergeordnete Instanzen (z.B. Kanton) ein Gesamtbild ergeben. Dieser Arbeitsschritt ist aber oft dem Einfluß der Region entzogen.

In der Realisierungsphase eines Projektes (Planauflage, Bereinigung) kann die Region dem Träger Alternativen anbieten, die zur Optimierung des Vorhabens beitragen.

Die Region muß in erster Linie ihre Grundsätze vertreten, aber bei einer Realisierung entgegen ihrem Willen trotzdem in der Lage sein, Alternativen und Varianten zu diskutieren, Gespräche zu führen. Sie ist deshalb für die Führung der politischen Auseinandersetzung (z. B. Aktionskomitees) nicht geeignet.

\section{Literatur}

ATELIER 5 (1973): Planungsverband Region Burgdorf, Genereller Gesamtplan, Bern.

ATELIER 5 (1976): Planungsverband Region Burgdorf, Regionale Richtpläne, Textteil, Bern.

ATELIER 5 (1977): Planungsverband Region Burgdorf, Richtpläne, Bern.

EMCH + BERGER AG (1979): SBB Grauholzlinie, Projekt 1979. Bern.

EMCH + BERGER AG (1982): SBB Grauholzlinie, Projekt 1979/82, Bern.

EMCH + BERGER AG (1980): SBB Neue Haupttransversale West-Ost, Generelles Projekt 1980, Bern.

GROSJEAN, G. (1966/70): Planungsverband Region Burgdorf, Planungsunterlagen, Bern.

GÜLLER/INFRAS (1983): Zweckmäßigkeitsprüfung der neuen Eisenbahn-Haupttransversalen (NHT), Zürich.

GVK-CH (1977): 'Gesamtverkehrskonzeption Schweiz, Schlußbericht, Bern.

RUTSCHMANN, W. (1979): Schweizerische Bundesbahnen, Streckenausbau Rothrist-Hindelbank, Generelles Projekt 1978/79, Adliswil/Bern. 